\title{
Educational Virtual Environment Based on Oculus Rift and Leap Motion Devices
}

\author{
Matea Zilak \\ University of Zagreb, Faculty of \\ Electrical Engineering and \\ Computing \\ Zagreb, Croatia \\ matea.zilak@fer.hr
}

\author{
Zeljka Car \\ University of Zagreb, Faculty of \\ Electrical Engineering and \\ Computing \\ Zagreb, Croatia \\ zeljka.car@fer.hr
}

\author{
Gordan Jezic \\ University of Zagreb, Faculty of \\ Electrical Engineering and \\ Computing \\ Zagreb, Croatia \\ gordan.jezic@fer.hr
}

\begin{abstract}
Virtual reality (VR) technology offers numerous benefits in different application areas, especially in education. VR brings new approaches to learning that can make the education process more attractive, while at the same time learners can develop creativity and innovativeness. Despite the possible benefits that VR can offer, the use of VR is still not widespread for educational purposes. Furthermore, the potential of VR for assistive technologies in the Augmentative and Alternative Communication (AAC) domain is recognized but has not been fully exploited. In this paper, development of an elementary mathematical virtual classroom prototype based on Oculus Rift and Leap Motion devices is described. The learning concept used in the prototype was taken from the state-of-the-art AAC application for mobile devices that introduces children with the concept of quantity which is one of the preconditions for adopting the concept of number. To analyze user's satisfaction with the application and acceptance of a new technology in general, user evaluation of the developed prototype was conducted. In general, a positive feedback from users without disabilities suggests that it makes sense to combine VR elements with education as well as AAC technologies. Contributing factors, such as the level of immersion in VR environments, unnatural behavior of virtual hands, and the level of familiarity with the VR technology, are identified as some of the most important aspects that need to be considered in the follow-up studies concerning users with disabilities (i.e. children with complex communication needs).
\end{abstract}

\section{Keywords}

Human Computer Interaction; Virtual Reality; Educational; Oculus Rift; Leap Motion; User Evaluation; AAC

\section{INTRODUCTION}

Within the last few years Virtual Reality (VR) technology has experienced growth of its popularity which had an impact on development of VR applications for practical use in areas other than entertainment and gaming, such as education. Much research has already been conducted on the application of VR in education which revealed numerous benefits that VR offers in this area [Pan10]. Traditional teaching and learning methods require little or no interaction with a student which makes them very static and, consequently, student's attention cannot be kept for a long time [Ray16]. On the other hand, VR requires interaction and encourages active participation rather than passivity, which has an

Permission to make digital or hard copies of all or part of this work for personal or classroom use is granted without fee provided that copies are not made or distributed for profit or commercial advantage and that copies bear this notice and the full citation on the first page. To copy otherwise, or republish, to post on servers or to redistribute to lists, requires prior specific permission and/or a fee. impact on increased level of motivation of students towards learning [Pan10].

Youngblut in [You98] presented unique capabilities of VR technology, such as the ability to visualize abstract concepts, to observe events at atomic or planetary scales, and to provide teaching in virtual environments that are impossible to visualize in physical classroom due to different safety, distance or time factors. Because of many examples of abstract problems that it provides, mathematics is an area in which VR can help learners to visualize abstract mathematical facts and understand problems that require, for example, spatial skills which are sometimes hard to understand for students [Kau09]. An extensive study on the educational applications of VR conducted by authors in [Mik11] revealed that majority of empirical studies they reviewed refer to science and mathematics topics which suggests that these areas offer a number of challenges that can be addressed with the use of VR technology.

Even though VR systems are now more acceptable and affordable than before, there are still people who have never experienced it and, therefore, never used it 
for educational purposes. Other types of educational technologies are used instead, such as smart boards, long-distance learning and mobile learning. Since the use of virtual reality is still not widespread, we have been motivated to propose a prototype of elementary mathematical virtual classroom with which a user is able to interact. In particular, human computer interaction in this prototype is based on the mechanism by which the user can interact in an intuitive way, with his/her hands. The objective behind the development of this kind of VR application is to analyze a new method of learning, based on modern technology such as virtual reality, and its acceptance.

The authors in [Dun12] described benefits that virtual worlds can have in teaching and learning, as they can provide socialization, entertainment and a laboratory for collaborative work. However, they emphasized that the use of virtual worlds for education should not disadvantage particular social, minority or disabled groups. According to [Lig07], technologies such as VR educational and play environments are an example of assistive technologies that may offer children with complex communication needs $(\mathrm{CCN})$ the means to interact positively with nondisabled peers (e.g. the classmates, neighbors, potential friends of children with $\mathrm{CCN}$ ) on an equal footing. That way, children with disabilities can overcome significant attitudinal barriers they confront with in society and can increase their self-esteem and self-empowerment [Lig07]. Augmentative and Alternative Communication (AAC) technologies support the communication process of persons with CCN. As a part of a research group which conducts intensive research in the AAC domain over nine years, especially within the EU funded ICT-AAC project [Ict13], we have developed over 30 AAC applications for most popular platforms, such as Android, iOS and Web. The idea behind the development of the applications was to make the learning process and communication as attractive as possible to encourage users to use the applications. For our AAC applications to be accessible and highly usable, in development process of every AAC application, as described in [Bab15], we cooperated with experts from different fields, such as rehabilitation and education and graphic design. The research in the AAC field is also analyzed from a technical point of view where technology capabilities regarding Machine-to-Machine communication are investigated. To properly interpret information about the user and his environment, communication and interoperability of different systems are necessary, so machine social networks [Pti16] impose as a possible solution for AAC systems to adapt as much as possible to the user. Example of a system like this can be a VR environment which provides a context to support social interaction for children with CCN. Since the potential of VR technology in AAC domain has not quite been exploited [Lig07], we have been additionally motivated to explore possible benefits that VR can bring in the AAC domain. Although our AAC applications, which are mostly tablet-based, have a stable and growing user base [Bab15], we are intrigued to cope with a challenge in developing VRbased AAC applications that can make a difference for children with CCN.

Considering previously written and the fact that there is a wide range of potential uses of VR in education, especially in mathematical domains for younger students as well as university level students [Kau09], a VR application that provides experience of learning basic mathematical concepts for younger children can be considered as a convenient option for the first step in the process of introducing VR for educational purposes (to a wider mass). That is why the prototype we proposed was developed based on one of the AAC applications developed within the ICT-AAC project. In the prototype we developed, the Oculus Rift headset is used for immersion into the virtual world while the Leap Motion sensor device is used for hand tracking. To analyze user satisfaction with usage of the VR application we developed and acceptance of the use of new technology in general, user evaluation is conducted and documented within this paper.

The paper is organized as follows: Section II details related work, Section III describes the AAC application on which development of the prototype was based, Section IV brings the description of the system architecture, implementation and functionalities, the process and the results of user evaluation are described in the Section V, and Section VI concludes the paper and presents a few ideas for future work.

\section{RELATED WORK}

Many VR environments for various educational purposes can be found. In [You98] Youngblut described many solutions developed for various educational purposes. One of the notable solutions is MaxwellWorld, an example of application developed as a research tool that provides a fully immersive and multisensory interface. Students interact with the virtual environment using virtual hands and menus (a Polhemus 3-Ball device [Ded96] is used for selection of menu item). Evaluation of the use of MaxwellWorld resulted in finding some important characteristics that aided learning, such as 3D representations, the interactivity, the ability to navigate to multiple perspectives, and the use of color. 
In addition to that, when compared to the EM Field ${ }^{1}$ computer-based simulator, MaxwellWorld is rated as easier to understand (due to better representations), but as harder to use (due to troubles while using the 3Ball and virtual hand) [Ded96]. As students differ in their interaction styles, interaction with virtual environment should be intuitive, understandable and well-known. User interface with those characteristics is called a Natural User Interface (NUI) and includes the use of devices that enable such interaction, such as Leap Motion [Lin16].

Educational virtual environment based on visualization of procedures and abstract concepts positively influences learning, especially if the user communicates with the virtual world in the form of an interactive game. That way, the user learns efficiently, but in an entertaining way, as the authors described in [Gri16] to be the case with the innovative educational environment for learning search algorithms, a topic which is often considered challenging for students to master.

Example of a virtual environment that uses key elements of successful computer games and emotionally appealing graphics is $\mathrm{SMILE}^{2}$ (Science and Math in an Immersive Learning Environment), one of the first bilingual immersive virtual learning environments for deaf and hearing students. The user interacts with virtual characters using the sign language by learning mathematical concepts and mathematical terminology of the American Sign Language (ASL). Formative evaluation of the game showed that the children perceived the game as more fun and easier to use and slightly more challenging than expected [Ada07].

Not many VR applications where the Oculus Rift and the Leap Motion are exclusively used can be found in literature, especially for educational purposes, but some of them can be identified as notable uses. The authors in [Lin16] described a VR system where the Oculus Rift and the Leap Motion are used. The purpose of a system they developed is to facilitate the selection of scientific articles which can be useful to researchers in their work. They proposed a new interface in which user interacts with his/her own hands and voice to enable natural interaction. Authors investigated different interaction techniques for immersive virtual environments including selection techniques and concluded that some of them are less intuitive than others, e.g. manipulation by gestures is less intuitive than direct manipulation in which

\footnotetext{
1 EM Field by D. Trowbridge and B. Sherwood, http://www.physics.umd.edu/rgroups/ripe/software/emfie ld.html

${ }^{2}$ SMILE, http://hpcg.purdue.edu/idealab/smile/about.html
}

selection of objects by virtual hand is identical as it is in the physical world [Lin16].

VR application the authors described in [Ala17] is developed to solve some of the educational problems which are still present among students, such as the lack of student's attention and difficulties to visualize what is being taught. Furthermore, virtual environment in which different experiments can be done is provided to avoid injuries that might occur in real environment because of an improperly conducted experiment. Also, this application enables easier performance of experiments to the disabled students because the use of VR headset and motion controller helps them to avoid movement struggles they usually have [Ala17].

After the literature survey on VR technology application areas, we specified the following for the elementary mathematical virtual classroom prototype:

- $\quad$ for the prototype to be easily understandable to children, natural interaction and intuitive selection technique will be achieved with the use of Leap Motion device, and

- for the prototype to be appealing to children, learning will be realized through an interactive game augmented with appropriate graphics.

\section{ROLE-MODEL APPLICATION}

As mentioned earlier, development of the prototype was based on one of the AAC applications developed within the ICT-AAC project. The application chosen to be the role-model application in this work is the ICT-AAC Domino counter ${ }^{3}$ mobile application. It is an application for mobile devices (smartphones, tablets) with Android or iOS operating systems. This application provides the children with developmental disabilities early experiences with definition of quantity and numbers in an easy and attractive way, enriched with appropriate images and sound recordings. Knowledge of quantity is one of the prerequisites for adopting the concept of number and basis for future calculation. In addition to this, use of ICT-AAC Domino counter goes from the use in family environment and/or pre-school institutions to use in the initial stage of math teaching in elementary schools. Although ICT-AAC Domino counter is primarily intendent for children with disabilities, the application can also be used by young children of typical development where there is no need for additional professional support [Ict14]. For these reasons, ICT-AAC Domino counter is considered as a suitable application to be the role-model when

3 ICT-AAC Domino counter on Google Play Store, https://play.google.com/store/apps/details?id=hr.fer.ztel.i ctaac.domino_brojalica 
developing the prototype of elementary mathematical virtual classroom.

\section{Learning Concept of ICT-AAC Domino Counter Application}

The application helps users to learn about the quantity by using the so-called domino principle - by linking a certain number of symbols with a corresponding number of dots on domino tiles. Within the application, users can learn about quantity by counting symbols or by recognizing the given number. At the beginning, the user is offered to choose between four possible game levels (learning to three, five, seven or ten) and, after that, between two possible game modes (playing with numbers or symbols). Depending on which game mode the user has chosen, tasks in the game are displayed by numbers or by symbols which the user needs to associate with appropriate answers displayed in the form of domino tiles. Home screen of the ICT-AAC Domino counter application is shown in Figure 1. The application has settings in which it is possible to choose between different options to customize the interface (e.g. choose between growing and mixed order of the domino tiles, select the number of tasks displaying in one round as well as the number of answers (domino tiles)).

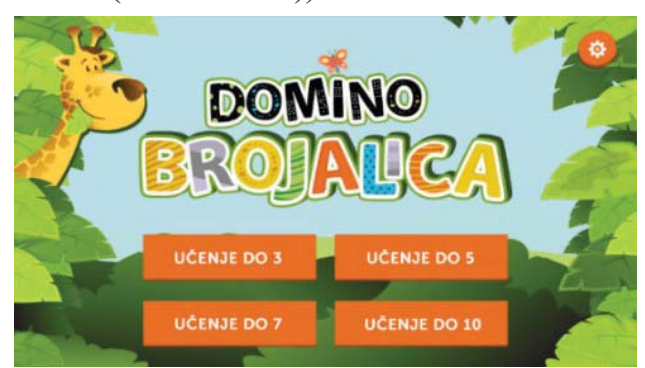

Figure 1 Home screen of the ICT-AAC Domino counter application

\section{Serious Game Design Elements in ICT- AAC Domino Counter Application}

In addition to being intended for younger children and enhancing the early math literacy skills required for later understanding of basic calculation, the ICT-AAC Domino counter application already has several game design elements that enhance the efficiency of educational tools and which can be utilized when implementing the prototype of an elementary mathematical virtual classroom. The importance of game design elements is explained by authors in [Ada07], who defined several game design elements that promote motivation of children to play the game again, enjoyment, and, therefore, learning. These elements are: a clearly defined background story and an overall structure of the game that gives meaning to all the activities of the game, the overall goal of the game, virtual world represented in a visual style that is appealing to the target age group (for children it is cartoon-like), multiple levels with variable difficulty, rewards associated with advancement, tips instead of answers. Besides the game design elements, design of interaction also has a role in encouraging user to continue playing - increased possibility of interaction with virtual world has a positive influence. That being said, some of the features of the ICT-AAC Domino counter are as follows:

- a graphical user interface presented in a style that is appealing to the target age group,

- multiple levels with variable difficulty,

- prominent progress through the game and

- feedback on the correctly/incorrectly answered task in visual and acoustic form.

\section{MATHEMATICAL VIRTUAL CLASSROOM PROTOTYPE}

\section{System Architecture}

An overview of the system architecture of the Mathematical Virtual Classroom is shown in Figure 2. One can notice that there is a two-way interaction between a user and the system. Firstly, the user's head and hand movements are monitored by two input units of the system: i) Leap Motion (i.e. sensor that tracks hands movements) and ii) Oculus camera (i.e. camera that tracks user's headset movement). Input data, such as head tracking data (e.g. headset's orientation and position) and hand tracking data (e.g. palm's and fingers' position and direction), are then transferred to the computer which runs the VR application developed within the Unity3D game engine. The retrieved data is then processed in real time and the appropriate simulation of the virtual environment and user's virtual hands is generated. Rendered image of the 3D world is then displayed on two output units at the same time: i) the Oculus Rift head-mounted display and ii) a diagnostic screen. Furthermore, audio output from the application is sent to the speaker which is responsible for giving the user audio feedback depending on user's actions during the game (e.g. positive audio feedback for a correct answer).

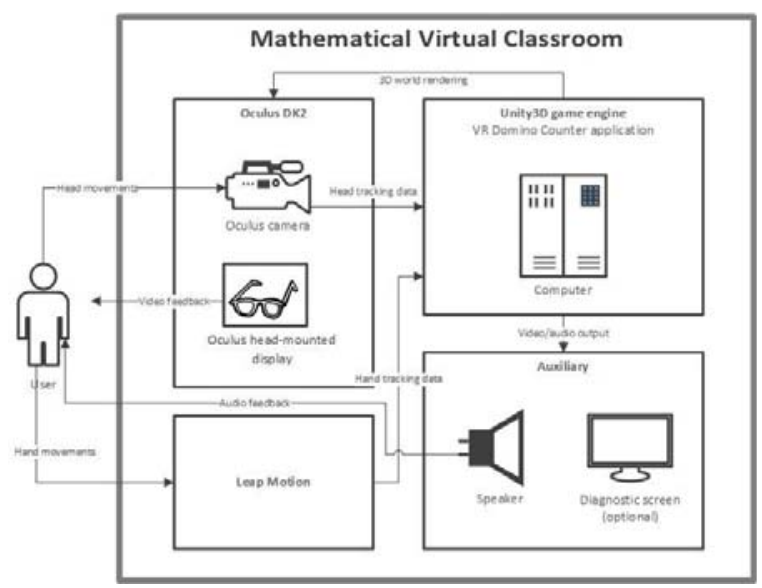

Figure 2 The system architecture of the Mathematical Virtual Classroom 


\section{Assets Used in the Mathematical Virtual Classroom}

For development of the mathematical virtual classroom prototype, the Unity game engine (version 5.5.2f1) was used. To enable immersion into and interaction with virtual environment, the Oculus Rift and Leap Motion modules are integrated with Unity software. Unity has built-in VR support for the Oculus Rift and the Leap Motion. The Oculus offers optional utilities including different scripts, prefabs, and other resources to assist with development. For development of the mathematical virtual classroom based on the ICT-AAC Domino counter application, resources from the Oculus Utilities 1.3.2 package were used. To develop VR application using the Leap Motion, it was needed to retrieve the Leap Motion Orion Beta software for development. In our project the Leap Developer Kit 3.2.0 was used. Additionally, to access the classes and functions offered by the Leap Motion Application Program Interface (API), Unity needs to include the Unity Core Assets basic package. In our project the Leap Motion Orion Beta 4.2.0 package was used. Extensions like the Hands Module 2.1.2 and the UI Input Module 1.2.1 are used to facilitate development of user interface and design of hands models.

Besides assets mentioned above, other assets from the official Unity asset store and online stores of 3D models were used to display terrain, the background of the scene and various objects in the virtual environment such as trees, wooden panel on which different UI elements are shown, wooden table on which domino tiles as answers appear, domino tile models etc. Figure 3 shows what user sees in the virtual environment when he/she is in the middle of the terrain with extended hands in front of him.

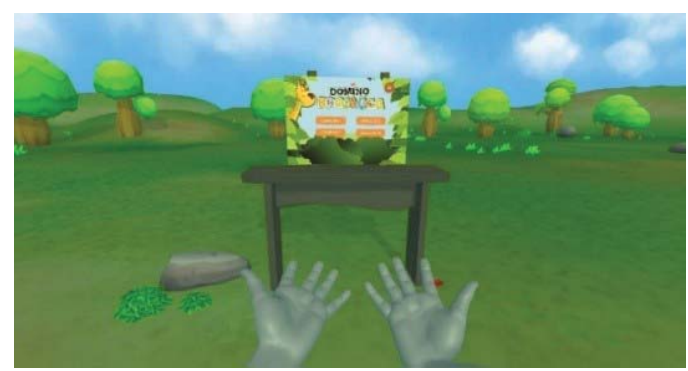

Figure 3 User's view from the middle of the terrain

\section{Basic Functionalities}

Because prototype development was based on the ICT-AAC Domino counter application, it was necessary to realize most of the functionalities that the ICT-AAC Domino counter has. The flow of the VR application is the same as the flow of the Domino counter application in general - at the beginning the user selects one of four possible game levels and one of two possible game modes followed by displaying problematic tasks in the form of numbers or symbols that user needs to associate with appropriate domino tiles. It is also possible for a user to change some settings, e.g. change the number of tasks or answers displaying in one round and change the order of domino tiles displaying (growing or mixed order).

In the ICT-AAC Domino counter application, the user interacts with the system by using simple gestures such as pushing buttons displayed on the touchscreen, while in the mathematical virtual classroom the user is interacting with virtual objects with his/her (virtual) hands. The interaction technique used includes selection of objects by virtual hand - for selection of game levels, game modes and problematic tasks displayed on the panel, appropriate gesture such as pushing buttons is used. In order to link certain task with appropriate answer, user needs to touch the 3D domino tile model displayed on the table.

Figure 4 shows what user sees when choosing between playing with numbers or symbols. An example of the task displayed by numbers is shown in Figure 5 while an example of the task displayed by symbols is shown in Figure 6. An example of a game moment when the task is answered incorrectly is shown in Figure 7 while an example of correctly answered task is shown in Figure 8 .

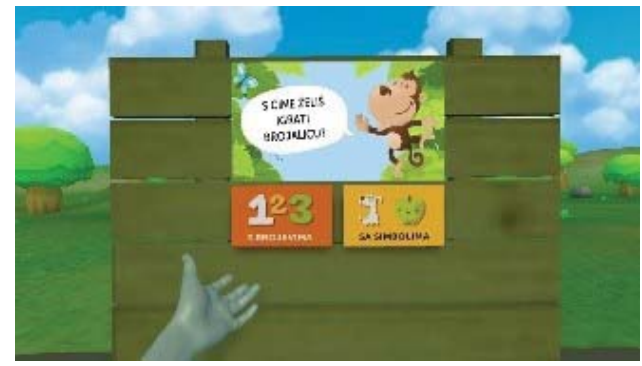

Figure 4 User's view when choosing the game

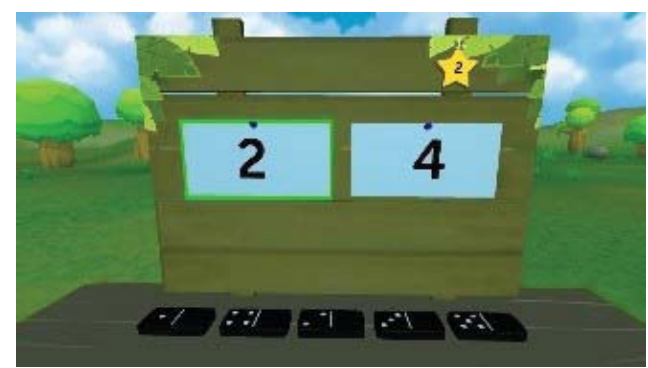

Figure 5 Task example displayed by numbers

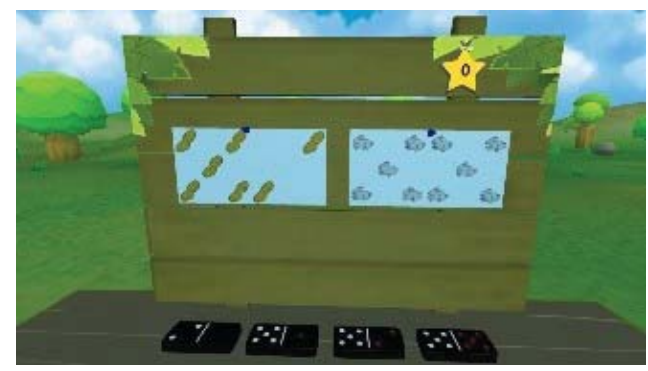

Figure 6 Task example displayed by symbols 


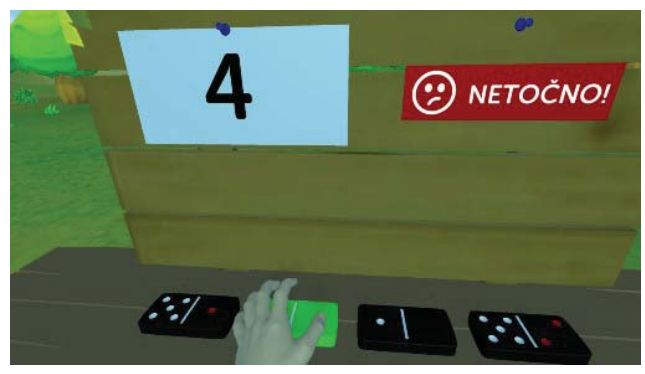

Figure 7 Example of a task answered incorrectly

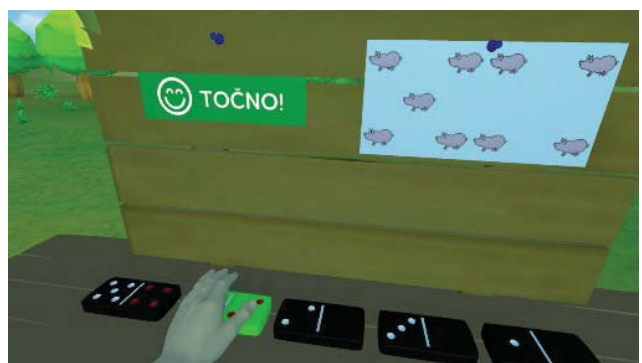

Figure 8 Example of a task answered correctly

\section{MATHEMATICAL VIRTUAL CLASSROOM USER EVALUATION}

To conduct user evaluation of the developed mathematical virtual classroom prototype, subjective and objective measures were specified. Objective measures related to the game play time and the number of incorrect answers were implemented in the software application itself while subjective measures of user satisfaction were collected through anonymous questionnaires after the application was used. The prototype was evaluated on a sample of 30 students of different gender and age. The login feature was also added to be able to distinguish measures for each user.

\section{Setup for the Experiment}

To use the elementary mathematical virtual classroom properly, it is necessary to do next: the VR application needs to be run on the computer while the user needs to put the Oculus Rift with the Leap Motion attached on his/her head. In addition, it is necessary to ensure that the camera is placed in the appropriate position to track the user's head position (in this case camera should be placed on the top and the center of the monitor). Also, the user should be sitting during interaction to reduce the possibility for motion sickness (otherwise, it is possible for user to play a game in a standing position as well as to explore the environment walking around on a distance that is permitted by the cables). By launching an application, the user is immersed into the virtual environment that is displayed on the head-mounted display. At the beginning, each participant entered his/her username using the virtual keyboard and after that, the user was introduced with how the application works by selecting one of three game levels: learning to 3,5 or 7. After successfully completing the selected game, the participant activated the measurement of objective measures by selecting the game level "Learning to 10 ". Figure 9 shows a user using the application while wearing the Oculus Rift with attached Leap Motion.

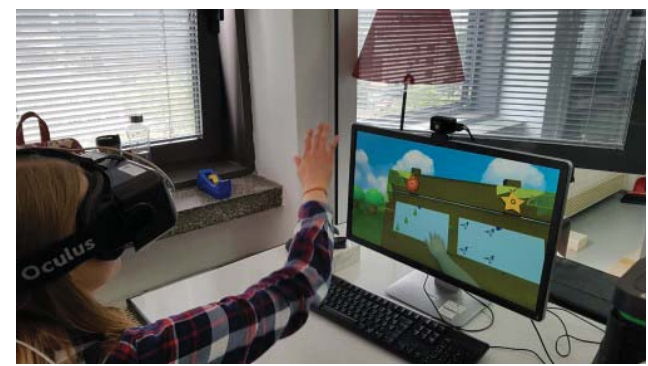

Figure 9 User interacts with virtual world objects

\section{Results of Objective Measures}

Since the prerequisite for successful use of the VR application is that the user is familiar with the concept of using virtual hands to interact in the virtual environment, for the purposes of user evaluation, the Expert is defined as a user who is experienced in using the VR application. Table 1 shows the comparison of the average playing time of the participants and the playing time of the Expert. As it can be seen from Table 1, the participants' average playing time needed for successful completion of the game is 2.5 times greater than the playing time of the Expert.

\begin{tabular}{|l|c|c|}
\hline User & Participant & Expert \\
\hline Average playing time $[s]$ & 58.02 & 23.19 \\
\hline
\end{tabular}

Table 1 Comparison of participants' average playing time and playing time of the Expert

None of the participants had a shorter game play time than the Expert which was expected. Also, there is a noticeable difference between the results of each participant. The shortest playing time of the participants is 26.88 seconds, which makes it only about 3 seconds slower than the time of the Expert, while the longest playing time is 101.23 seconds. Total of 11 participants out of 30 (about $36 \%$ ) had at least one incorrect answer during the game. It was expected that the participants who had a greater number of incorrect answers will have a longer playing time, but as it can be seen from the graph shown in Figure 10, there is no significant correlation between the duration of time participant spent in-game and number of incorrect answers. These results indicate that the performance of the game depends on how the individual has accepted immersion into the virtual world and the way of interacting with the virtual world, and how the individual felt while using this type of technology. 


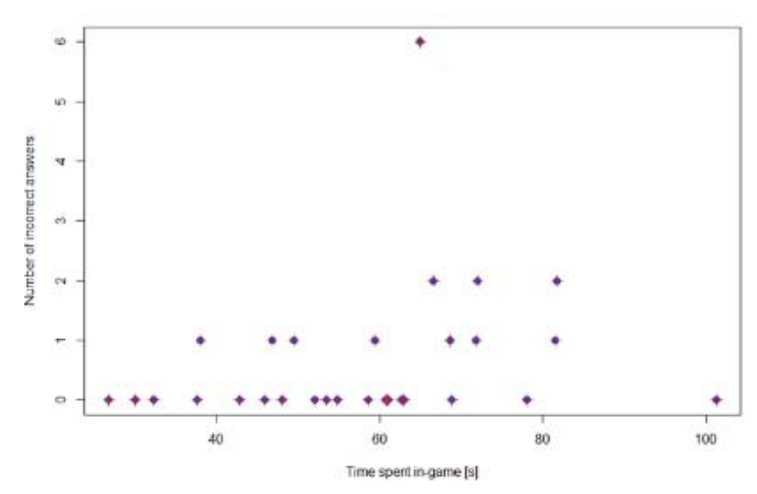

Figure 10 Correlation between time spent in-game and number of incorrect answers

\section{Results of Subjective Measures}

To collect subjective measures of satisfaction, each participant approached the questionnaire after he/she used the application. The ratings (marks) given for every question fall within a Likert scale, where the mark " 1 " is interpreted as "strongly disagree" and " 5 " is interpreted as "strongly agree". Table 2 shows seven questions used in the user satisfaction questionnaire and average mark calculated for each question. On the fifth question participant answered only if he/she had at least one incorrect answer.

\begin{tabular}{|c|l|c|}
\hline $\begin{array}{c}\text { Question } \\
\text { Number }\end{array}$ & \multicolumn{1}{|c|}{ Question } & $\begin{array}{c}\text { Avg. } \\
\text { Mark }\end{array}$ \\
\hline 1 & The application was easy to use. & 4.93 \\
\hline 2 & $\begin{array}{l}\text { I was feeling comfortable while } \\
\text { using the application. }\end{array}$ & 4.8 \\
\hline 3 & $\begin{array}{l}\text { I am satisfied with the speed of the } \\
\text { application. }\end{array}$ & 4.93 \\
\hline 4 & $\begin{array}{l}\text { Interaction with user interface } \\
\text { elements (e.g. buttons, domino } \\
\text { tiles) was intuitive. }\end{array}$ & 4.9 \\
\hline 5 & $\begin{array}{l}\text { Incorrect answers were the result } \\
\text { of intentionally choosing the } \\
\text { wrong answer (rather than } \\
\text { unnatural behavior of virtual } \\
\text { hands). }\end{array}$ & 4 \\
\hline 6 & $\begin{array}{l}\text { I like the principle of immersion } \\
\text { in the virtual world and the } \\
\text { possibility of interaction with } \\
\text { virtual hands. }\end{array}$ & 4.87 \\
\hline 7 & $\begin{array}{l}\text { Overall, I am satisfied with the } \\
\text { application. }\end{array}$ & 5 \\
\hline
\end{tabular}

Table 2 Questions from the user satisfaction questionnaire and their average mark

The participants answered the last question unanimously with mark 5, meaning they are generally satisfied with the application. Because participants had little or no previous experience with VR, their satisfaction probably stems from the fact that they have not had the opportunity to experience VR in this way, where the interaction with the user interface and game objects is done in a natural and intuitive way (with virtual hands following the movements of their hands). This can also be seen from very high average marks regarding application ease of use (4.93), speed (4.93) and intuitive interaction with UI elements (4.9). A little lower average mark, but still very high, is related to the subjective feelings of the user, that is, the feeling of comfort during the use of the application (4.8) and the feeling of liking the principle of immersion into the virtual world and the possibility of interaction with virtual hands (4.87). These slightly lower marks are probably because the use of VR is still not widespread, so users are still not used to the feeling of full immersion into the virtual environment.

The lowest average mark (4.00) is calculated for the question regarding tasks that were answered incorrectly. Participants were supposed to answer whether inaccurate answer(s) were the result of intentional selection of the answer that was wrong or unnatural behavior of virtual hands. This question was asked because sometimes it may happen that the information obtained from the Leap Motion sensors is wrongly interpreted. This is most commonly occurring when the application is started or when some other object in the background engages in an area where the position of the hand is tracked. If this happened at the time a user needed to answer, it could be the reason why the user unintentionally answered incorrectly. Of all the participants who answered the fifth question only three of them "blamed" the application, i.e. the unnatural behavior of the virtual hands, which means that the wrong interpretation positioning of the hand position does not occur frequently.

The graph in Figure 11 shows average mark calculated from marks that participants gave for each question. Participants who gave average marks lower than 4.5 are the ones who gave mark 1 for the fifth question, but duration of their game play was shorter than average play time. Two participants who gave marks lower than the average $(<4.7)$ but greater than 4.5 had longer playing time than average. These results (lower marks from participants who experienced unnatural behavior of virtual hands and who had longer game play time than average) indicate a certain correlation between the results from objective and subjective measures. The rest of the participants gave average marks greater than 4.7 , and their playing time varied (shorter and longer than average). Interesting fact to note is that 12 participants out of the $30(40 \%)$ gave the average mark equal to 5 , and that group of participants includes both the person with the shortest and the person with the longest playing time. From this we can conclude that the reason the people who gave great marks played the game for a long time was not due to problems during the game but because they liked the feeling of immersion in the virtual world, so they prolonged the interaction. 


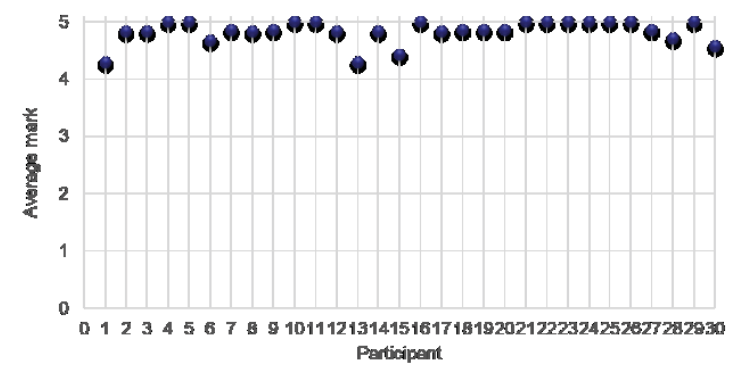

Figure 11 Graphical illustration of participants' average marks for research questions

\section{CONCLUSIONS AND FUTURE WORK}

As many researchers established, education is an area where VR technology can contribute to learning and teaching methods in a different way than traditional methods. These methods require interaction with a student by which he/she can develop creativity and innovativeness. In addition, an area which provides a lot of examples of abstract problems and where the VR potential can be utilized is mathematics. Because the use of VR is still not widespread, especially for educational purposes, the prototype of the elementary mathematical virtual classroom was developed. It is based on one of the ICT-AAC applications for learning basic mathematical concepts for younger children. That said, such an application does not require much skill or effort regarding spatial awareness as it is just a first step towards introducing VR for educational purposes to a wider mass. Prototype is then evaluated to analyze users' satisfaction with the application and the acceptance of the use of new technology in general. Learning in virtual environment in a natural and intuitive way provided by mathematical virtual classroom prototype goes beyond methods in formal education which require little or no interaction with students.

The results of user evaluation of mathematical virtual classroom showed that significant correlation between the duration of playing time and learning outcomes (in this case expressed as answers for a given mathematical problem task) does not exist but that the performance of a game depends exclusively on how an individual liked the immersion into and interaction in the virtual world. On the other hand, a certain correlation occurred between the results from objective and subjective measures for some participants. Participants who gave lower marks experienced unnatural behavior of virtual hands and had longer game play time than average. Other participants gave great marks even though they played the game for a long time which is an indicator that they enjoyed the virtual world and had no problems during the game. In general, the results of user evaluation showed overall satisfaction with the application which stems from the attractiveness of using modern technology. The results also showed that users are still not completely accustomed to the feeling of immersion into the virtual environment which stems from the fact that the concept of using virtual reality is still new. That is why further research is needed to make VR solutions as intuitive and understandable as possible in different domains.

For mathematical virtual classroom to be adopted as AAC system that children will be motivated to use, future research needs to be conducted. The initial user evaluation was conducted on participants without disabilities and the results were positive. Because of that, we have basis for further work where we plan to test the application with typically developing children as well as with children with disabilities to investigate their preferences and to see how they would accept the concept of learning in the virtual environment.

Encouraging feedback from the conducted study, which involved a mathematical application with a simple VR environment, reveals a potential for tackling new interesting research challenges in the follow-up study. For example, to be able to measure the possible impact of VR technologies in education, especially in mathematics domain, one can develop a more sophisticated VR application, intended for understanding and solving mathematical problems, that include spatial context. Also, future work will include an extensive user experience analysis of both versions of the application (i.e., the "traditional" mobile version and the VR version). Such an analysis will benchmark the two different ways of hand interaction mechanics: i) through a touchscreen (i.e. touch), and ii) through VR elements (e.g. grabbing and pointing). Ultimately, the analysis will shed a light on whether the VR systems are more engaging and more intuitive than the traditional methods of learning.

\section{ACKNOWLEDGMENTS}

This work has been supported by Croatian Science Foundation under the project 8813 (Human-centric Communications in Smart Networks of People, Machines and Organizations) and by Croatian Regulatory Authority for Network Industries under the project Looking to the Future 2020.

\section{REFERENCES}

[Ada07] Adamo-Villani, N. and Wright, K. (2007). SMILE: An immersive learning game for deaf and hearing children. In ACM SIGGRAPH 2007 educators program (SIGGRAPH '07). ACM, New York, NY, USA, Article 17

[Ala17] AlAwadhi, S., AlHabib, N. and Murad, D. Virtual reality application for interactive and informative learning. 2017 2nd International Conference on Bioengineering for Smart Technologies (BioSMART), Paris, 2017, pp. 1-4.

[Bab15] Babic, J., Slivar, I., Car, Z. and Podobnik, V. Prototype-driven software development process for 
augmentative and alternative communication applications. $201513^{\text {th }}$ International Conference on Telecommunications (ConTEL), Graz, 2015, pp. 1-8.

[Ded96] Dede, C., Salzman, M., and Loftin, B. (1996). MaxwellWorld: Learning complex scientific concepts via immersion in virtual reality. In Proceedings of the 2nd International Conference on Learning Sciences, pp. 22-29.

[Dun12] Duncan I., Miller, A., Jiang, S.: A taxonomy of virtual worlds usage in education. British Journal of Educational Technology, 43, 6 (2012), 949-964.

[Gri16] Grivokostopoulou, F., Perikos, I. and Hatzilygeroudis, I. An Innovative Educational Environment Based on Virtual Reality and Gamification for Learning Search Algorithms. IEEE 8th International Conference on Technology for Education (T4E), Mumbai, India, 2016, str. 110-115

[Ict13] ICT-AAC project. (2013). ICT competence network for innovative services for persons with complex communication needs (ICT-AAC). Retrieved from: http://www.ict-aac.hr/index.php/en/

[Ict14] ICT-AAC Domino counter. (2014). Retrieved from: http://www.ict-aac.hr/index.php/hr/ict-aac-razvijeneaplikacije/android-aplikacije/domino-brojalica

[Kau09] Kaufmann, H. Virtual environments for mathematics and geometry education. Themes in Science and Technology Education, Special Issue: Virtual Reality in Education, vol. 2 (2009), 1-2; 131-152

[Lig07] Light, J., Page, R., Curran, J. and Pitkin, L. (2007). Children's ideas for the design of AAC assistive technologies for young children with complex communication needs. In Augmentative and Alternative Communication, 23, 4: 274-287.

[Lin16] Linares, R., Herrera, J. and Alfaro, L., "AliciaVR: Exploration of scientific articles in an immersive virtual environment with natural user interfaces," 2016 IEEE Ecuador Technical Chapters Meeting (ETCM), Guayaquil, 2016, pp. 1-6.

[Mik11] Mikropoulos, T. A., Natsis, A.: Educational virtual environments: A ten-year review of empirical research (1999-2009). Computers \& Education 56, 3 (2011), 769 -780 .

[Pan10] Pantelidis, V. S. (2010). Reasons to use virtual reality in education and training courses and a model to determine when to use virtual reality. Themes in Science and Technology Education, 2(1-2), 59-70.

[Pti16] Pticek, M., Podobnik, V. and Jezic, G. (2016). "Beyond the Internet of Things: The Social Networking of Machines", International Journal of Distributed Sensor Networks, pp. 1-15

[Ray16] Ray, A.B. and Deb, S. Smartphone based virtual reality systems in classroom teaching - a study on the effects of learning outcome. In 2016 IEEE 8th International Conference on Technology for Education (T4E)

[You98] Youngblut, C. (1998). Educational uses of virtual reality technology. Technical report, IDA Document $D$ 2128. Alexandria, VA. Institute for Defense Analyses 